CALT-68-2081

CMU-HEP96-14

UCSD-TH-96-26

hep-ph/9610518

\title{
Corrections to the Bjorken and Voloshin sum rules
}

\author{
C. Glenn Boyd ${ }^{a}$, Zoltan Ligeti ${ }^{b}$, Ira Z. Rothstein ${ }^{c}$, Mark B. Wise ${ }^{b}$ \\ ${ }^{a}$ Department of Physics, Carnegie Mellon University, Pittsburgh, PA 15213 \\ ${ }^{b}$ California Institute of Technology, Pasadena, CA 91125 \\ ${ }^{c}$ Department of Physics, University of California at San Diego, La Jolla, CA 92093
}

\begin{abstract}
We calculate near zero recoil the order $\alpha_{s}$ corrections to the Bjorken and Voloshin sum rules that bound the $B \rightarrow D^{(*)} \ell \bar{\nu}$ form factors. These bounds are derived by relating the result of inserting a complete set of physical states in a time ordered product of weak currents to the operator product expansion. The sum rules sum over physical states with excitation energies less than a scale $\Delta$. We find that the corrections to the Bjorken bound are moderate, while the Voloshin bound receives sizable corrections enhanced by $\Delta / \Lambda_{\mathrm{QCD}}$. With some assumptions, we find that the slope parameter for the form factor $h_{A_{1}}$ in $B \rightarrow D^{*} \ell \bar{\nu}$ decay satisfies $0.4 \lesssim \rho_{A_{1}}^{2} \lesssim 1.3$.
\end{abstract}




\section{INTRODUCTION}

It is possible to perform model independent extractions of some of the CKM matrix elements and quark masses from exclusive and inclusive semileptonic $B$ meson decays via a systematic expansion in inverse powers of the heavy bottom and charm quark masses. The form factors in $B \rightarrow D^{(*)} \ell \bar{\nu}$ decays are related by heavy quark symmetry [1] to the Isgur-Wise function, $\xi\left(w=v \cdot v^{\prime}\right)$, where $v$ is the four-velocity of the $B$ and $v^{\prime}$ is that of the $D^{(*)}$. A model independent determination of $\left|V_{c b}\right|$ from the differential decay rate $\mathrm{d} \Gamma\left(B \rightarrow D^{*} \ell \bar{\nu}\right) / \mathrm{d} w$ is made possible by the fact that $\xi$ is equal to unity at zero recoil $(w=1)$ [1 [4. Inclusive $B$ decay rates can be calculated by performing an operator product expansion for the time ordered product of two weak currents [5], allowing for a model independent determination of $\left|V_{c b}\right|$ from the inclusive semileptonic decay rate, $\Gamma\left(B \rightarrow X_{c} \ell \bar{\nu}\right)$.

The major theoretical uncertainties in the determination of $\left|V_{c b}\right|$ from inclusive decays are due to the questionable convergence of the perturbative corrections to the $b$ quark decay rate [6], and the uncertainties in the $b$ and $c$ quark masses. Uncertainties in the determination of $\left|V_{c b}\right|$ from $B \rightarrow D^{*} \ell \bar{\nu}$ originate from order $\Lambda_{\mathrm{QCD}}^{2} / m_{c, b}^{2}$ corrections at zero recoil, and from extrapolating the form factors measured at $w>1$ to $w=1$ (phase space vanishes at $w=1$ ). The uncertainties in this extrapolation would be reduced if the slope of the form factor at zero recoil were known.

Sum rules have been derived that relate the exclusive decay form factors to the inclusive decay rates. The Bjorken sum rule [7,8] gives the bound $\rho^{2}>1 / 4$, where $\rho^{2}$ is minus the slope of the Isgur-Wise function. Voloshin derived the upper bound $\rho^{2}<1 / 4+\left(m_{M}-\right.$ $\left.m_{Q}\right) /\left[2\left(m_{M_{1}}-m_{M}\right)\right] \simeq 0.75$ [9], where $m_{M}-m_{Q}=\bar{\Lambda}$ is the mass difference between the ground state heavy meson and the heavy quark that it contains (up to corrections of order $\left.\Lambda_{\mathrm{QCD}}^{2} / m_{c, b}^{2}\right)$, and $m_{M_{1}}-m_{M}$ is the mass of the first excited meson state above the pseudoscalar-vector doublet.

The most recent experimental data from CLEO [10] is $\rho_{B \rightarrow D^{*}}^{2}=0.84 \pm 0.12 \pm 0.08$. This might violate the above upper bound when experimental uncertainties decrease. The 
ALEPH [11] result $\rho_{B \rightarrow D^{*}}^{2}=0.29 \pm 0.18 \pm 0.12$ is significantly smaller, close to the above lower bound. The slope of the form factor $h_{A_{1}}$, which occurs in $B \rightarrow D^{*} \ell \bar{\nu}$ decay, has also been studied by CLEO [12]. Central values for its slope parameter $\rho_{A_{1}}^{2}$ ranging between 0.91 and 1.53 have been obtained. Thus, it is interesting to calculate the corrections to the Bjorken and Voloshin bounds.

\section{REVIEW OF SUM RULES}

To derive the sum rules, we follow Refs. $13 \sqrt{15}$. Consider the time-ordered product

$$
T_{\mu \nu}=\frac{i}{2 m_{B}} \int \mathrm{d}^{4} x e^{-i q \cdot x}\left\langle B\left|T\left\{J_{\mu}^{\dagger}(x), J_{\nu}(0)\right\}\right| B\right\rangle,
$$

where $J_{\mu}$ is a $b \rightarrow c$ axial or vector current, the $B$ states are at rest, $\vec{q}$ is fixed, and $q_{0}=m_{B}-E_{M}-\epsilon$. Here $E_{M}=\sqrt{m_{M}^{2}+|\vec{q}|^{2}}$ is the minimal possible energy of the hadronic final statef that can be created by the current $J_{\mu}$ with fixed $|\vec{q}|$. With this definition of $\epsilon$ in terms of the hadronic variables, the cut of $T_{\mu \nu}$ in the complex $\epsilon$ plane corresponding to physical states with a charm quark lies along $0<\epsilon<+\infty$. It will be important that at the same value of $|\vec{q}|$ the cut at the parton level lies within the smaller region $\epsilon>$ $\bar{\Lambda}(w-1) / w+\mathcal{O}\left(\Lambda_{\mathrm{QCD}}^{2} / m_{c, b}\right)$. ( $T_{\mu \nu}$ has another cut corresponding to physical states with two $b$ quarks and a $\bar{c}$ quark that lies between $-2 E_{M}>\epsilon>-\infty$. This cut will not be important for our discussion.) To separate out specific hadronic form factors, one contracts the currents in (1) with a suitably chosen four-vector $a$, yielding

$$
a^{* \mu} T_{\mu \nu}(\epsilon) a^{\nu}=\frac{1}{2 m_{B}} \sum_{X}(2 \pi)^{3} \delta^{3}\left(\vec{q}+\vec{p}_{X}\right) \frac{\left\langle B\left|J^{\dagger} \cdot a^{*}\right| X\right\rangle\langle X|J \cdot a| B\rangle}{E_{X}-E_{M}-\epsilon}+\ldots,
$$

where the ellipses denote the contribution from the cut corresponding to two $b$ quarks and a $\bar{c}$ quark. The sum over $X$ includes the usual phase space factors, i.e., $\mathrm{d}^{3} p / 2 E_{X}$ for each

\footnotetext{
${ }^{*}$ The ground state doublet of mesons have light degrees of freedom with spin-parity $s_{l}^{\pi_{l}}=\frac{1}{2}^{-}$. We consider situations when only one member of this doublet contributes. It is this state that we denote by $M$.
} 


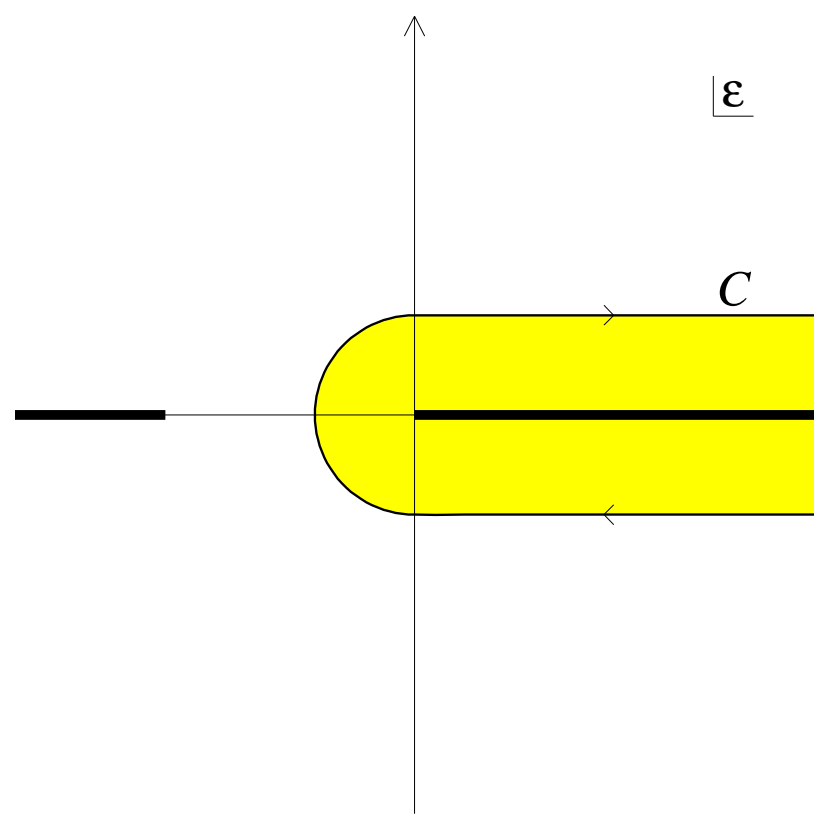

FIG. 1. The integration contour $C$ in the complex $\epsilon$ plane. The cuts extend to $\operatorname{Re} \epsilon \rightarrow \pm \infty$.

particle in the state $X$.

While $T_{\mu \nu}(\epsilon)$ cannot be computed for arbitrary values of $\epsilon$, its integrals with appropriate weight functions are calculable in perturbative QCD. Consider integration of the product of a weight function $W_{\Delta}(\epsilon)$ with $T_{\mu \nu}(\epsilon)$ along the contour $C$ surrounding the physical cut, shown in Fig. 1. Assuming $W$ is analytic in the shaded region enclosed by this contour, we get

$$
\frac{1}{2 \pi i} \int_{C} \mathrm{~d} \epsilon W_{\Delta}(\epsilon)\left[a^{* \mu} T_{\mu \nu}(\epsilon) a^{\nu}\right]=\sum_{X} W_{\Delta}\left(E_{X}-E_{M}\right)(2 \pi)^{3} \delta^{3}\left(\vec{q}+\vec{p}_{X}\right) \frac{|\langle X|J \cdot a| B\rangle|^{2}}{2 m_{B}} .
$$

The positivity of $|\langle X|J \cdot a| B\rangle|^{2}$ for all states $X$ gives an upper bound on the magnitude of form factors mediating $B$ decays into the ground state doublet $M$.

The integral of the correlator weighted with $\epsilon W_{\Delta}(\epsilon)$ eliminates the contribution from the ground state doublet $X=M$, yielding

$$
\begin{aligned}
& \frac{1}{2 \pi i} \int_{C} \epsilon \mathrm{d} \epsilon W_{\Delta}(\epsilon)\left[a^{* \mu} T_{\mu \nu}(\epsilon) a^{\nu}\right] \\
& =\sum_{X \neq M} W_{\Delta}\left(E_{X}-E_{M}\right)(2 \pi)^{3} \delta^{3}\left(\vec{q}+\vec{p}_{X}\right)\left(E_{X}-E_{M}\right) \frac{|\langle X|J \cdot a| B\rangle|^{2}}{2 m_{B}} .
\end{aligned}
$$


This can be turned into an upper bound on the contribution of excited states $(X \neq M)$ to the right-hand side of (3) by assuming that the contribution of multi-hadron states is negligible below the first excited meson state, $M_{1}$. This is true in the large $N_{c}$ limit, and experimental data available in the future on $B \rightarrow D^{(*)} \pi \ell \bar{\nu}$, etc., decay rates can support (or oppose) the validity of this assumption.

Thus, there are upper and lower bounds

$$
\begin{aligned}
\frac{1}{2 \pi i} \int_{C} \mathrm{~d} \epsilon W_{\Delta}(\epsilon)\left[a^{* \mu} T_{\mu \nu}(\epsilon) a^{\nu}\right] & >\frac{|\langle M|J \cdot a| B\rangle|^{2}}{4 m_{B} E_{M}} \\
& >\frac{1}{2 \pi i} \int_{C} \mathrm{~d} \epsilon W_{\Delta}(\epsilon)\left[a^{* \mu} T_{\mu \nu}(\epsilon) a^{\nu}\right]\left(1-\frac{\epsilon}{E_{M_{1}}-E_{M}}\right),
\end{aligned}
$$

where $E_{M_{1}}=\sqrt{m_{M_{1}}^{2}+|\vec{q}|^{2}}$. It should be emphasized that while this upper bound (which yields the Bjorken bound) is essentially model independent, the lower bound (which yields the Voloshin bound) relies on the above assumptions about the spectrum of the final state hadrons $X$.

Following [14], we choose a set of weight functions

$$
W_{\Delta}^{(n)}(\epsilon)=\frac{\Delta^{2 n}}{\epsilon^{2 n}+\Delta^{2 n}}, \quad(n=2,3, \ldots)
$$

that satisfy the following properties: $(i) W_{\Delta}$ is positive semidefinite along the cut so that every term in the sum over $X$ on the hadron side of the sum rule is non-negative; (ii) $W_{\Delta}(0)=1 ;(i i i) W_{\Delta}$ is flat near $\epsilon=0 ;(i v)$ and $W_{\Delta}$ falls off rapidly to zero for $\epsilon>\Delta$. This choice of weight functions is motivated by the fact that for values of $n$ of order unity all the poles of $W_{\Delta}^{(n)}$ lie at a distance of order $\Delta$ away from the physical cut. As $n \rightarrow \infty, W_{\Delta}^{(n)}$ approaches $\theta(\Delta-\epsilon)$ for $\epsilon>0$, which corresponds to summing over all hadronic resonances up to excitation energy $\Delta$ with equal weight. In this limit the poles of $W_{\Delta}^{(n)}$ approach the cut, and the contour $C$ is forced to lie within a distance of order $\Delta / n$ from the cut at $\epsilon=\Delta$. In this case the evaluation of the contour integrals using perturbative QCD relies on local duality [16] at the scale $\Delta$. In the rest of this paper whenever the weight function is not specified explicitly, we mean $\theta(\Delta-\epsilon)$. 


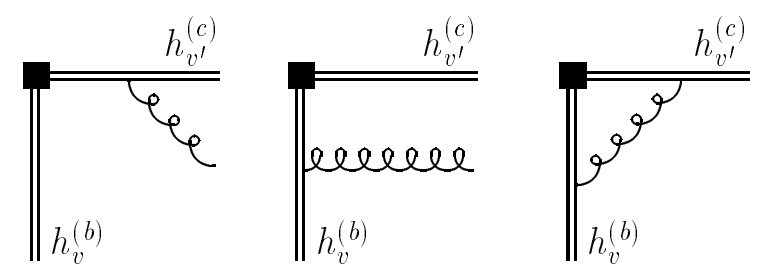

FIG. 2. Feynman diagrams that contribute to the order $\alpha_{s}$ corrections to the sum rules. The heavy quark fields in the effective theory are denoted by $h^{(c, b)}$. The black square indicates insertion of the $b \rightarrow c$ axial or vector current.

The bounds in eq. (5) become weaker as $\Delta$ is increased. However, the scale $\Delta$ must be chosen large enough that the contour integrals in eq. (5) can be performed using perturbative QCD, allowing the evaluation of the Wilson coefficients of the operators that occur in the operator product expansion for the time ordered product of currents. In practice this means that $\Delta$ must be greater than about $1 \mathrm{GeV}$.

\section{BOUNDS ON THE ISGUR-WISE FUNCTION}

The bounds stemming from eq. (5) are simplest to evaluate in the heavy quark effective theory (HQET) [17]. One may consider the vector current in the effective theory, $V^{\mu}=\bar{h}_{v^{\prime}}^{(c)} \gamma^{\mu} h_{v}^{(b)}$, and choose $a_{\mu}=v_{\mu}$, the four-velocity of the $B$. Instead of calculating the correlator itself, it is simpler to compute its imaginary part given by the diagrams in Fig. 2. In this paper we focus on the region near zero recoil, and therefore we expand the perturbative corrections to linear order in $(w-1)$.

The ground state contribution is $\left\langle M\left|V^{\mu} v_{\mu}\right| B\right\rangle=\sqrt{m_{B} m_{M}}(1+w) \xi(w)$, where $\xi(w)$ is the Isgur-Wise function. (Only the pseudoscalar member of the ground state doublet contributes to this matrix element.) In the $\overline{\mathrm{MS}}$ scheme, using dimensional regularization and a finite gluon mass, $m_{g}$, the inclusive expression for the correlator to order $\alpha_{s}$ reads

$$
\begin{aligned}
\frac{1}{\pi} \operatorname{Im}[T(\epsilon)]=\frac{1+w}{2 w}[ & \delta\left(\epsilon-\bar{\Lambda} \frac{w-1}{w}\right)+(w-1) \frac{8 \alpha_{s}}{9 \pi} \ln \frac{m_{g}^{2}}{\mu^{2}} \delta(\epsilon) \\
& \left.+(w-1) \frac{8 \alpha_{s}}{9 \pi} \frac{2 \epsilon^{2}+m_{g}^{2}}{\epsilon^{4}} \sqrt{\epsilon^{2}-m_{g}^{2}} \theta\left(\epsilon-m_{g}\right)\right]+\ldots
\end{aligned}
$$

In eq. (7) the terms proportional to delta functions come from the charm quark final state, 
and the term proportional to $\theta\left(\epsilon-m_{g}\right)$ arises from final states with a charm quark and a single gluon. The gluon mass is used to regulate an infrared divergence in the integral of $\operatorname{Im}[T(\epsilon)]$ over $\epsilon$, which cancels (at order $\alpha_{s}$ ) between these two types of final states.

Using eqs. (3) and (4) this implies the following sum rules

$$
\begin{aligned}
& \frac{1+w}{2 w}\left[1+(w-1) \frac{8 \alpha_{s}}{9 \pi}\left(\ln \frac{4 \Delta^{2}}{\mu^{2}}-\frac{5}{3}\right)\right]=\frac{(1+w)^{2}}{4 w}|\xi(w)|^{2}+\ldots \\
& \frac{1+w}{2 w}\left[1-\frac{\bar{\Lambda}(w-1)}{m_{M_{1}}-m_{M}}\left(1+\frac{16 \alpha_{s}}{9 \pi} \frac{\Delta}{\bar{\Lambda}}\right)\right. \\
& \left.\quad+(w-1) \frac{8 \alpha_{s}}{9 \pi}\left(\ln \frac{4 \Delta^{2}}{\mu^{2}}-\frac{5}{3}\right)\right]=\frac{(1+w)^{2}}{4 w}|\xi(w)|^{2}-\ldots
\end{aligned}
$$

The ellipses in these equations denote positive terms whose first derivatives at $w=1$ are also positive. The reason for positivity of the first derivative is that in the effective theory all excited state contributions must vanish at $w=1$, and may therefore be written as $(w-1)$ times the square of some form factor. (Eq. (8) was previously obtained using a Wilson line approach to heavy quark interactions in Ref. [18. See also 19.)

In eqs. (8), $\alpha_{s}$ is evaluated at the subtraction point $\mu$. This $\mu$-dependence on the lefthand side of eq. (8) is cancelled by the $\mu$-dependence of the Isgur-Wise function, which we define in the $\overline{\mathrm{MS}}$ scheme. Differentiating with respect to $w$, we find the following bounds on the slope parameter of the Isgur-Wise function, $\rho^{2}=-\mathrm{d} \xi(w) /\left.\mathrm{d} w\right|_{w=1}$,

$$
\frac{1}{4}+\frac{\bar{\Lambda}}{2\left(m_{M_{1}}-m_{M}\right)}+\frac{4 \alpha_{s}}{9 \pi}\left(\frac{2 \Delta}{m_{M_{1}}-m_{M}}+\frac{5}{3}-\ln \frac{4 \Delta^{2}}{\mu^{2}}\right)>\rho^{2}(\mu)>\frac{1}{4}+\frac{4 \alpha_{s}}{9 \pi}\left(\frac{5}{3}-\ln \frac{4 \Delta^{2}}{\mu^{2}}\right) .
$$

Neglecting the order $\alpha_{s}$ corrections, these are precisely the Bjorken and Voloshin bounds discussed in the introduction. The upper bound on $\rho^{2}$ receives a perturbative correction of order $\alpha_{s} \Delta / \Lambda_{\mathrm{QCD}}$, which is very large in the $\Delta \gg \Lambda_{\mathrm{QCD}}$ limit Note, however, that higher orders in perturbation theory do not produce additional powers of $\Delta / \Lambda_{\mathrm{QCD}}$. Similarly, sum

\footnotetext{
${ }^{\dagger}$ One is free to absorb all or part of this correction into a redefinition of $\bar{\Lambda}, \bar{\Lambda} \rightarrow \bar{\Lambda}(\Delta)$, provided one consistently reexpresses other formulae involving $\bar{\Lambda}$ in terms of this new quantity.
} 
rules involving higher moments of $\operatorname{Im}[T(\epsilon)]$ [20,13] will receive perturbative strong interaction corrections enhanced by more powers of $\Delta / \Lambda_{\mathrm{QCD}}$.

The bounds on the slope of the Isgur-Wise function in eq. (9) will have a perturbative series without large logarithms in its coefficients if the subtraction point $\mu$ is chosen to be equal to $\Delta$. Note that the second term in the upper bound, proportional to $\bar{\Lambda}$, has a renormalon ambiguity of order unity (since the heavy quark pole mass has a renormalon ambiguity of order $\left.\Lambda_{\mathrm{QCD}}\right)$. This is canceled by the ambiguity in the perturbative series in $\alpha_{s}$ that multiplies $\Delta /\left(m_{M_{1}}-m_{M}\right)$, the first term of which is presented in eq. (9).

Using a weight function $W_{\Delta}^{(n)}(\epsilon)$ other than $W_{\Delta}^{(\infty)}(\epsilon)=\theta(\Delta-\epsilon)$ does not affect the lower (Bjorken) bound on $\rho^{2}$ given on the right-hand side of eq. (9). Therefore, for the lower bound on $\rho^{2}$ (at order $\alpha_{s}$ ), using the weight function $W_{\Delta}^{(\infty)}(\epsilon)$ does not rely on the assumption of local duality at the scale $\Delta$. Such corrections, however, weaken the upper (Voloshin) bound by adding

$$
\frac{4 \alpha_{s}}{9 \pi} \frac{\Delta}{m_{M_{1}}-m_{M}}\left(\frac{\pi}{n \sin [\pi /(2 n)]}-2\right)
$$

to the left-hand side of eq. (9). Numerical estimates of these corrections will be given later.

So far we have focused on the perturbative corrections to the coefficient of the lowest dimension operator, $\bar{h}_{v}^{(b)} h_{v}^{(b)}$, that occurs in the operator product expansion for the time ordered product. Higher dimension operators are of the form $\bar{h}_{v}^{(b)}\left(v^{\prime} \cdot D\right)^{p} h_{v}^{(b)}$. These yield corrections suppressed by powers of $\Lambda_{\mathrm{QCD}} / \Delta$ for weight functions other than $W_{\Delta}^{(\infty)}(\epsilon)=$ $\theta(\Delta-\epsilon)$

A lower bound on $\rho^{2}$ including order $\alpha_{s}$ perturbative QCD corrections was derived in Ref. [21]. It corresponds to a weight function given by the phase-space of $b$ decay, which is different from those considered here. The bound in [21] appears stronger than that in eq. (9) because the weight function given by the phase-space falls off faster with $\epsilon$.

To zeroth order in $\alpha_{s}$ and $\Lambda_{\mathrm{QCD}} / m_{c, b}$, the constraints in the effective theory are identical with bounds on the slope of the measured shape of the $B \rightarrow D^{(*)} \ell \bar{\nu}$ decay spectra. However, at first order in $\alpha_{s}$, one has to combine the above results with corrections that originate from 
matching the full theory onto the heavy quark effective theory. [This will also eliminate the $\mu$-dependence from the bounds in eq. (9).]

\section{DIFFERENTIAL DECAY RATES}

We are interested in the form factors of semileptonic $B \rightarrow D^{(*)} \ell \bar{\nu}$ decays, defined as

$$
\begin{aligned}
& \frac{\left\langle D\left(v^{\prime}\right)\left|V^{\mu}\right| B(v)\right\rangle}{\sqrt{m_{D} m_{B}}}=h_{+}(w)\left(v+v^{\prime}\right)^{\mu}+h_{-}(w)\left(v-v^{\prime}\right)^{\mu}, \\
& \frac{\left\langle D^{*}\left(v^{\prime}\right)\left|V^{\mu}\right| B(v)\right\rangle}{\sqrt{m_{D^{*}} m_{B}}}=i h_{V}(w) \varepsilon^{\mu \nu \alpha \beta} \epsilon_{\nu}^{*} v_{\alpha}^{\prime} v_{\beta}, \\
& \frac{\left\langle D^{*}\left(v^{\prime}\right)\left|A^{\mu}\right| B(v)\right\rangle}{\sqrt{m_{D^{*}} m_{B}}}=h_{A_{1}}(w)(w+1) \epsilon^{* \mu}-h_{A_{2}}(w)\left(\epsilon^{*} \cdot v\right) v^{\mu}-h_{A_{3}}(w)\left(\epsilon^{*} \cdot v\right) v^{\prime \mu} .
\end{aligned}
$$

Here $V^{\mu}=\bar{c} \gamma^{\mu} b$ and $A^{\mu}=\bar{c} \gamma^{\mu} \gamma_{5} b$ are the vector and axial currents. The kinematic variable $w$ is related to $q^{2}$ via $w=\left(m_{B}^{2}+m_{D^{(*)}}^{2}-q^{2}\right) /\left(2 m_{B} m_{D^{(*)}}\right)$. Up to corrections suppressed by powers of $\alpha_{s}\left(m_{c, b}\right)$ and $\Lambda_{\mathrm{QCD}} / m_{c, b}, h_{-}(w)=h_{A_{2}}(w)=0$ and $h_{+}(w)=h_{V}(w)=h_{A_{1}}(w)=$ $h_{A_{3}}(w)=\xi(w)$, where the Isgur-Wise function, $\xi(w)$, is evaluated at a subtraction point around $m_{c, b}$.

Experimentally the differential decay rates are measured, and are usually quoted in terms of the functions $\mathcal{F}_{B \rightarrow D^{(*)}}(w)$, defined below. At tree level, and without $\Lambda_{\mathrm{QCD}} / m_{c, b}$ corrections, these functions are identical to the Isgur-Wise function, so their slopes are equal to that of $\xi(w)$. However, at order $\alpha_{s}$ additional corrections beyond those calculated in eq. (9) using the effective theory arise from matching the full QCD onto the HQET. Corrections suppressed by powers of $\Lambda_{\mathrm{QCD}} / m_{c, b}$ arise from higher dimension operators in the HQET Lagrangian, and from higher dimension current operators in the effective theory.

With the above definitions of the form factors, and $r^{(*)}=m_{D^{(*)}} / m_{B}$, the differential decay rates are

$$
\begin{aligned}
\frac{\mathrm{d} \Gamma\left(B \rightarrow D^{*} \ell \bar{\nu}\right)}{\mathrm{d} w} & =\frac{G_{F}^{2} m_{B}^{5}}{48 \pi^{3}} r^{* 3}\left(1-r^{*}\right)^{2}\left(w^{2}-1\right)^{1 / 2}(w+1)^{2} \\
& \times\left[1+\frac{4 w}{w+1} \frac{1-2 w r^{*}+r^{* 2}}{\left(1-r^{*}\right)^{2}}\right]\left|V_{c b}\right|^{2}\left|\mathcal{F}_{B \rightarrow D^{*}}(w)\right|^{2}, \\
\frac{\mathrm{d} \Gamma(B \rightarrow D \ell \bar{\nu})}{\mathrm{d} w} & =\frac{G_{F}^{2} m_{B}^{5}}{48 \pi^{3}} r^{3}(1+r)^{2}\left(w^{2}-1\right)^{3 / 2}\left|V_{c b}\right|^{2}\left|\mathcal{F}_{B \rightarrow D}(w)\right|^{2} .
\end{aligned}
$$


The functions $\mathcal{F}_{B \rightarrow D^{*}}$ and $\mathcal{F}_{B \rightarrow D}$ are given in terms of the form factors of the vector and axial currents defined in (11) as

$$
\begin{aligned}
\left|\mathcal{F}_{B \rightarrow D^{*}}(w)\right|^{2}=\left[1+\frac{4 w}{w+1} \frac{1-2 w r^{*}+r^{* 2}}{\left(1-r^{*}\right)^{2}}\right]^{-1}\left\{\frac{1-2 w r^{*}+r^{* 2}}{\left(1-r^{*}\right)^{2}} 2\left[h_{A_{1}}^{2}(w)+\frac{w-1}{w+1} h_{V}^{2}(w)\right]\right. \\
\left.\quad+\left[h_{A_{1}}(w)+\frac{w-1}{1-r^{*}}\left(h_{A_{1}}(w)-h_{A_{3}}(w)-r^{*} h_{A_{2}}(w)\right)\right]^{2}\right\} \\
\mathcal{F}_{B \rightarrow D}(w)=h_{+}(w)-\frac{1-r}{1+r} h_{-}(w) .
\end{aligned}
$$

We define the "physical" slope parameters, $\rho_{B \rightarrow D^{*}}^{2}$ and $\rho_{B \rightarrow D}^{2}$, via

$$
\begin{aligned}
\left|\mathcal{F}_{B \rightarrow D^{*}}(w)\right| & =\left|\mathcal{F}_{B \rightarrow D^{*}}(1)\right|\left[1-\rho_{B \rightarrow D^{*}}^{2}(w-1)+\ldots\right], \\
\left|\mathcal{F}_{B \rightarrow D}(w)\right| & =\left|\mathcal{F}_{B \rightarrow D}(1)\right|\left[1-\rho_{B \rightarrow D}^{2}(w-1)+\ldots\right] .
\end{aligned}
$$

Note that $\mathcal{F}_{B \rightarrow D^{*}}(1)=h_{A_{1}}(1)$. Due to Luke's theorem [四] $h_{A_{1}}(1)=\eta_{A}+\mathcal{O}\left(\Lambda_{\mathrm{QCD}}^{2} / m_{c, b}^{2}\right)$, while $\mathcal{F}_{B \rightarrow D}(1)=\eta_{V}+\mathcal{O}\left(\Lambda_{\mathrm{QCD}} / m_{c, b}\right)$. The quantities $\eta_{A}$ and $\eta_{V}$ relate the axial and vector currents in the full theory of QCD to those in HQET at zero recoil.

The order $\alpha_{s}$ corrections to the relationship between $\rho_{B \rightarrow D^{(*)}}^{2}$ and the Isgur-Wise function can be computed model independently. We combine the results of the previous section with the order $\alpha_{s}$ matching corrections [22] taken from Ref. [23] to derive bounds on the slope parameters. Denoting $z=m_{c} / m_{b}$, and approximating $r^{(*)} \simeq z$ in the order $\alpha_{s}$ corrections, the slope of the Isgur-Wise function is related to that of $\mathcal{F}_{B \rightarrow D^{(*)}}$ via

$$
\rho_{B \rightarrow D^{(*)}}^{2}=\rho^{2}(\mu)+\frac{4 \alpha_{s}}{9 \pi} \ln \frac{m_{c}^{2}}{\mu^{2}}+\frac{\alpha_{s}}{\pi}\left(\delta_{B \rightarrow D^{(*)}}^{\left(\alpha_{s}\right)}-\frac{20}{27}\right)+\frac{\bar{\Lambda}}{2 m_{c}} \delta_{B \rightarrow D^{(*)}}^{(1 / m)} .
$$

Using eq. (9) this implies the bounds

$$
\begin{aligned}
& \rho_{B \rightarrow D^{(*)}}^{2}>\frac{1}{4}+\frac{4 \alpha_{s}}{9 \pi} \ln \frac{m_{c}^{2}}{4 \Delta^{2}}+\frac{\alpha_{s}}{\pi} \delta_{B \rightarrow D^{(*)}}^{\left(\alpha_{s}\right)}+\frac{\bar{\Lambda}}{2 m_{c}} \delta_{B \rightarrow D^{(*)}}^{(1 / m)}, \\
& \rho_{B \rightarrow D^{(*)}}^{2}<\frac{1}{4}+\frac{\bar{\Lambda}}{2\left(m_{M_{1}}-m_{M}\right)}\left(1+\frac{16 \alpha_{s}}{9 \pi} \frac{\Delta}{\bar{\Lambda}}\right)+\frac{4 \alpha_{s}}{9 \pi} \ln \frac{m_{c}^{2}}{4 \Delta^{2}}+\frac{\alpha_{s}}{\pi} \delta_{B \rightarrow D^{(*)}}^{\left(\alpha_{s}\right)}+\frac{\bar{\Lambda}}{2 m_{c}} \delta_{B \rightarrow D^{(*)}}^{(1 / m)} .
\end{aligned}
$$

The $\Delta$-independent part of the order $\alpha_{s}$ corrections is contained in $\delta_{B \rightarrow D^{(*)}}^{\left(\alpha_{s}\right)}$, while $\delta_{B \rightarrow D^{(*)}}^{(1 / m)}$ contains the order $\Lambda_{\mathrm{QCD}} / m_{c, b}$ corrections to $\rho_{B \rightarrow D^{(*)}}^{2}$. We find 


$$
\begin{aligned}
\delta_{B \rightarrow D^{*}}^{\left(\alpha_{s}\right)} & =\frac{2(1-z)\left(11+2 z+11 z^{2}\right)+24\left(2-z+z^{2}\right) z \ln z}{27(1-z)^{3}}, \\
\delta_{B \rightarrow D}^{\left(\alpha_{s}\right)} & =\frac{2(1-z)\left(23-34 z+23 z^{2}\right)+12\left(3-3 z+2 z^{2}\right) z \ln z}{27(1-z)^{3}} .
\end{aligned}
$$

The corrections in $\delta_{B \rightarrow D^{(*)}}^{(1 / m)}$ depend on the four subleading Isgur-Wise functions [四] that parametrize all first order deviations from the infinite mass limit. These can only be estimated at present using model predictions. Using the notation of [23] we find

$$
\begin{aligned}
\delta_{B \rightarrow D^{*}}^{(1 / m)}= & -2 \chi_{1}^{\prime}(1)+4 \chi_{3}^{\prime}(1)-z\left[2 \chi_{1}^{\prime}(1)-4 \chi_{2}(1)+12 \chi_{3}^{\prime}(1)\right] \\
& -\frac{5}{6}(1+z)-\frac{4}{3} \chi_{2}(1)-\frac{1-2 z+5 z^{2}}{3(1-z)} \eta(1), \\
\delta_{B \rightarrow D}^{(1 / m)}= & -(1+z)\left[2 \chi_{1}^{\prime}(1)-4 \chi_{2}(1)+12 \chi_{3}^{\prime}(1)\right]+\frac{2(1-z)^{2}}{1+z} \eta^{\prime}(1) .
\end{aligned}
$$

Here prime denotes $\mathrm{d} / \mathrm{d} w$.

Note that the bounds in eqs. (16) do not rely on the assumption that $m_{c, b} \gg \Delta$. The bounds on the slope of the Isgur-Wise function in HQET (where $m_{c, b} \rightarrow \infty$ ) hold as long as $\Delta$ is large enough for perturbative QCD to be a valid way to calculate the contour integrals in eq. (5). The values for the charm and bottom quark masses only arise in matching $\rho_{B \rightarrow D^{(*)}}^{2}$ onto the slope of the Isgur-Wise function.

There are several scales that occur in the bounds on $\rho_{B \rightarrow D^{(*)}}^{2}$. In the limit $m_{c, b} \gg \Delta$ we know how to sum the large logarithms of the ratio of scales $m_{c, b} / \Delta$ that occur in the perturbative corrections to the bounds. First one performs the matching of $\rho_{B \rightarrow D^{(*)}}^{2}$ onto the slope of the Isgur-Wise function at a scale around $m_{c, b}\left(e . g ., \sqrt{m_{c} m_{b}}\right)$, then one scales the Isgur-Wise function down from this subtaction point to $\Delta$ using the anomalous dimension for the operator $\bar{h}_{v^{\prime}}^{(c)} \gamma^{\mu} h_{v}^{(b)}$. Finally one applies the bound on the slope of the Isgur-Wise function in eq. (9) at the subtraction point $\mu=\Delta$. However, since $\Delta$ must be greater than $1 \mathrm{GeV}$, for the physical values of the charm and bottom quark masses $m_{c, b} / \Delta$ is not very large. Consequently, we did not sum the leading logarithms of this ratio using this renormalization group procedure in eqs. (16). 


\begin{tabular}{l||cc|cc}
\hline \hline & \multicolumn{2}{|c|}{ Bjorken bound $\left(\rho_{B \rightarrow D^{(*)}}^{2}>\ldots\right)$} & \multicolumn{2}{c}{ Voloshin bound $\left(\rho_{B \rightarrow D^{(*)}}^{2}<\ldots\right)$} \\
& $B \rightarrow D^{*}$ & $B \rightarrow D$ & $B \rightarrow D^{*}$ & $B \rightarrow D$ \\
\hline$\Delta=1 \mathrm{GeV}$ & 0.24 & 0.33 & 0.95 & 1.05 \\
$\Delta=2 \mathrm{GeV}$ & 0.18 & 0.27 & 1.11 & 1.20 \\
\hline \hline
\end{tabular}

TABLE I. Upper and lower bounds on $\rho_{B \rightarrow D^{(*)}}^{2}$, the slopes of the functions $\mathcal{F}_{B \rightarrow D^{(*)}}(w)$ at zero recoil, that describe the shape of the semileptonic $B \rightarrow D^{(*)} \ell \bar{\nu}$ decay spectrum. The order $\alpha_{s}$ corrections are included, while order $\Lambda_{\mathrm{QCD}} / m_{c, b}$ corrections are neglected. To zeroth order in $\alpha_{s}$ the Bjorken bound is 0.25 , while the Voloshin bound is 0.75 .

To evaluate the bounds in eq. (16) we takef $\alpha_{s}=0.3$ (corresponding to a scale of about $2 \mathrm{GeV}), \bar{\Lambda}=m_{M_{1}}-m_{M}=0.4 \mathrm{GeV}, m_{c}=1.4 \mathrm{GeV}$, and $m_{b}=4.8 \mathrm{GeV}$. However, since we neglect corrections of order $\alpha_{s}\left(\Lambda_{\mathrm{QCD}} / m_{c, b}\right)$, other values for the heavy quark masses, e.g. $m_{c} \simeq \bar{m}_{D}=\left(m_{D}+3 m_{D^{*}}\right) / 4=1.97 \mathrm{GeV}$ and $m_{b} \simeq \bar{m}_{B}=\left(m_{B}+3 m_{B^{*}}\right) / 4=5.31 \mathrm{GeV}$, would be equally valid. In Table I we show the Bjorken and Voloshin bounds at order $\alpha_{s}$ using $\Delta=1 \mathrm{GeV}$ and $\Delta=2 \mathrm{GeV}$, for the weight function $W_{\Delta}^{(\infty)}=\theta(\Delta-\epsilon)$. To obtain these numerical results we neglected $\delta_{B \rightarrow D^{(*)}}^{(1 / m)}$. Using $W_{\Delta}^{(2)}$ instead of $W_{\Delta}^{(\infty)}$ does not affect the Bjorken bound, but weakens the Voloshin bound by 0.02 for $\Delta=1 \mathrm{GeV}$, and by 0.05 for $\Delta=2 \mathrm{GeV}$. While the Bjorken bound only receives moderate corrections to its tree-level value of 0.25 , the corrections to the tree-level value of the Voloshin bound, 0.75 , are more sizable and strongly $\Delta$-dependent.

To estimate the possible size of the order $\Lambda_{\mathrm{QCD}} / m_{c, b}$ corrections in eq. (18), we use the QCD sum rule predictions for the four subleading universal functions [24]. These give approximately $\chi_{1}^{\prime}(1)=0.3, \chi_{2}(1)=-0.04, \chi_{3}^{\prime}(1)=0.02, \eta(1)=0.6$, and $\eta^{\prime}(1)=0$. Note that the results for $\delta_{B \rightarrow D^{(*)}}^{(1 / m)}$ depend sensitively on $\chi_{1}^{\prime}(1)$, which is only calculated to order $\alpha_{s}^{0}$ in the framework of QCD sum rules; the other subleading form factors $\left[\chi_{2}(w), \chi_{3}(w)\right.$, and

\footnotetext{
$\ddagger$ Unless explicitly stated otherwise, we use these values throughout this paper.
} 
$\eta(w)]$ are computed to order $\alpha_{s}$. We find that these corrections reduce $\rho_{B \rightarrow D^{*}}^{2}$ by 0.3 and $\rho_{B \rightarrow D}^{2}$ by 0.2 . However, these values are model dependent, and the uncertainties are large.

Using eq. (15) we find that the order $\alpha_{s}$ perturbative corrections predict that $\rho_{B \rightarrow D}^{2}$ is about 0.09 larger than $\rho_{B \rightarrow D^{*}}^{2}$. This prediction is affected by order $\Lambda_{\mathrm{QCD}} / m_{c, b}$ corrections [see eq. ([18)], and therefore it is not model independent. However, the QCD sum rule results

for the subleading Isgur-Wise functions predict that $\rho_{B \rightarrow D}^{2}-\rho_{B \rightarrow D^{*}}^{2}$ is further increased. Therefore, an enhancement of $\rho_{B \rightarrow D}^{2}$ compared to $\rho_{B \rightarrow D^{*}}^{2}$ by about $0.1-0.2$ seems quite likely, and a precise measurement of this difference would test the predictions of Ref. [24]. (Similar results were obtained in 25].)

As the above model estimates for the order $\Lambda_{\mathrm{QCD}} / m_{c, b}$ corrections are fairly sizable, one should investigate whether more reliable bounds can be derived in the full theory. The motivation is that in the full theory one can bound the magnitude of the physical form factors (equal to the Isgur-Wise function plus order $\Lambda_{\mathrm{QCD}} / m_{c, b}$ corrections in HQET). In the next section we derive bounds on the $h_{A_{1}}(w)$ form factor in the full theory. For comparison, we give here the bounds on this form factor in the effective theory approach. The slope of $h_{A_{1}}(w)$ at $w=1, \rho_{A_{1}}^{2}$, satisfies a bound of the same form as that in eq. (16). The order $\alpha_{s}$ corrections to the bounds on $\rho_{A_{1}}^{2}$ are

$$
\delta_{A_{1}}^{\left(\alpha_{s}\right)}=\frac{2(1-z)\left(17-4 z+17 z^{2}\right)+6\left(9-3 z+4 z^{2}\right) z \ln z}{27(1-z)^{3}},
$$

while the order $\Lambda_{\mathrm{QCD}} / m_{c, b}$ terms are

$$
\delta_{A_{1}}^{(1 / m)}=-2 \chi_{1}^{\prime}(1)+4 \chi_{3}^{\prime}(1)-z\left[2 \chi_{1}^{\prime}(1)-4 \chi_{2}(1)+12 \chi_{3}^{\prime}(1)\right]-\frac{1+z}{2}+z \eta(1) .
$$

Neglecting the corrections of order $\Lambda_{\mathrm{QCD}} / m_{c, b}$ this gives $0.28<\rho_{A_{1}}^{2}<0.99$ for $\Delta=1 \mathrm{GeV}$ and $0.22<\rho_{A_{1}}^{2}<1.15$ for $\Delta=2 \mathrm{GeV}$.

\section{SUM RULES IN THE FULL THEORY}

In the full theory, bounds on the form factor $h_{A_{1}}(w)$ in eq. (11) can be obtained from eq. (5) by taking the axial current and choosing the four-vector $a$ such that $a \cdot v=a \cdot q=0$. 
These bounds are expected to be less model dependent at present than those derived in the effective theory, as there is no uncertainty associated with the subleading Isgur-Wise functions (they contribute at order $\Lambda_{\mathrm{QCD}} / m_{c, b}$ in the effective theory approach). On the other hand, the bounds derived in the full theory receive calculable corrections proportional to powers of $\Delta / m_{c, b}$ at order $\alpha_{s}$, which do not arise in the HQET approach.

The $\Delta$-dependent part of the corrections away from zero recoil are straightforward to compute by considering the (finite) difference between the bremsstrahlung graphs in the full theory and in HQET. Since we want to keep the full $w$ dependence at order $\Lambda_{\mathrm{QCD}} / m_{c, b}$, we need to include

$$
\left(\frac{1+w}{2 w}\right)_{\text {parton }}=\left(\frac{1+w}{2 w}\right)_{\text {hadron }}\left[1-\frac{(w-1)}{w^{2}} \frac{\bar{\Lambda}}{m_{c}}+\ldots\right]
$$

Neglecting terms of order $\alpha_{s}^{2}, \Lambda_{\mathrm{QCD}}^{2} / m_{c, b}^{2}, \alpha_{s}\left(\Lambda_{\mathrm{QCD}} / m_{c, b}\right)$, and $\alpha_{s}(w-1)^{2}$, we obtain the bounds

$$
\begin{aligned}
& \frac{(1+w)^{2}\left|h_{A_{1}}(w)\right|^{2}}{4 w}<\frac{1+w}{2 w}\left[1-\frac{(w-1)}{w^{2}} \frac{\bar{\Lambda}}{m_{c}}\right]+\frac{\alpha_{s}}{\pi}[A+(w-1) X] \\
& \frac{(1+w)^{2}\left|h_{A_{1}}(w)\right|^{2}}{4 w}>\frac{1+w}{2 w}\left[1-\frac{(w-1)}{w^{2}} \frac{\bar{\Lambda}}{m_{c}}-\frac{V}{E_{M_{1}}-E_{M}}\right]+\frac{\alpha_{s}}{\pi}[B+(w-1) Y] .
\end{aligned}
$$

Here $A, B, X$, and $Y$ are functions of $m_{c}, m_{b}$, and $\Delta$. The term $V$ arises from the difference in the start of the parton and hadron cuts, and from matrix elements of dimension-5 terms in the operator product expansion. It is simple to extract from [13,15,

$$
V=(w-1)\left(\frac{\bar{\Lambda}}{w}+\frac{\bar{\Lambda}^{2}}{2 m_{c}} \frac{3-w}{w^{3}}+\frac{\lambda_{1}}{6 m_{c}} \frac{3+w}{w^{3}}+\frac{\lambda_{1}+3 \lambda_{2}}{3 m_{b}} \frac{1}{w}\right)+\ldots
$$

where

$$
\begin{aligned}
& \lambda_{1}=\frac{1}{2 m_{B}}\left\langle B(v)\left|\bar{h}_{v}^{(b)}(i D)^{2} h_{v}^{(b)}\right| B(v)\right\rangle, \\
& \lambda_{2}=\frac{1}{6 m_{B}}\left\langle B(v)\left|\bar{h}_{v}^{(b)} \frac{g}{2} \sigma_{\mu \nu} G^{\mu \nu} h_{v}^{(b)}\right| B(v)\right\rangle .
\end{aligned}
$$

Since $E_{M_{1}}-E_{M}$ is of order $\Lambda_{\mathrm{QCD}}$, all terms in $V$ contribute at least of order $\Lambda_{\mathrm{QCD}} / m_{c, b}$ to the lower bound on $\left|h_{A_{1}}(w)\right|^{2}$. We cannot neglect these terms, as the main motivation for 
considering the bounds in the full theory was to eliminate the order $\Lambda_{\mathrm{QCD}} / m_{c, b}$ uncertainties related to the subleading Isgur-Wise functions in the HQET approach.

The function $A$ was computed in [14] and $B$ was computed in 15]. Denoting $d=\Delta / m_{c}$, the result is

$$
\begin{gathered}
A=-2\left(\frac{1+z}{1-z} \ln z+\frac{8}{3}\right)+\frac{d(2+d)\left[2 z^{2}(1+d)^{2}-\left(3+2 z+z^{2}\right)\right]}{18(1+d)^{2}}+\frac{3+2 z-z^{2}}{9} \ln (1+d), \\
B=A-\frac{\Delta}{m_{M_{1}}-m_{M}}\left[\frac{\left(2+3 d+2 d^{2}\right)\left(9+6 z-3 z^{2}\right)+2 d^{2} z^{2}\left(8+7 d+2 d^{2}\right)}{54(1+d)^{2}}\right. \\
\left.-\frac{3+2 z-z^{2}}{9} \frac{\ln (1+d)}{d}\right] .
\end{gathered}
$$

We find that the coefficients $X$ and $Y$ are

$$
\begin{aligned}
X= & \left(\frac{1+z}{1-z} \ln z+\frac{8}{3}\right)+\frac{8}{9} \ln \left(4 d^{2}\right)-2 \delta_{A_{1}}^{\left(\alpha_{s}\right)}-\frac{d\left(16+42 d+45 d^{2}+16 d^{3}\right)}{9(1+d)^{4}} \\
& -\frac{2 d z\left(20+52 d+53 d^{2}+18 d^{3}\right)}{45(1+d)^{4}}+\frac{d z^{2}\left(12+52 d+71 d^{2}+44 d^{3}+10 d^{4}\right)}{45(1+d)^{4}} \\
& -\frac{80+12 z^{2}}{45} \ln (1+d), \\
Y= & X-\frac{\bar{\Lambda} A}{m_{M_{1}}-m_{M}}-\frac{\Delta}{m_{M_{1}}-m_{M}}\left\{\frac{16}{9}+\frac{6-11 d-62 d^{2}-83 d^{3}-32 d^{4}}{18(1+d)^{4}}\right. \\
& +\frac{z\left(38+113 d+122 d^{2}+41 d^{3}\right)}{45(1+d)^{4}}-\frac{z^{2}\left(34+119 d+134 d^{2}+49 d^{3}-16 d^{4}-10 d^{5}\right)}{90(1+d)^{4}} \\
& \left.-\frac{15+38 z-17 z^{2}}{45} \frac{\ln (1+d)}{d}\right\} .
\end{aligned}
$$

In Fig. 3 we plot the upper and lower bounds on $(1+w)^{2}\left|h_{A_{1}}(w)\right|^{2} /(4 w)$ over the region $1<w<1.25$, using eq. (22). Over this region of $w$, corrections of order $\alpha_{s}(w-1)^{2}$ that we have not computed are expected to be negligible. The thin and thick solid curves correspond to the choices $\Delta=1 \mathrm{GeV}$ and $2 \mathrm{GeV}$, respectively. The dashed curves show the upper and lower bounds neglecting the order $\alpha_{s}$ and $\Lambda_{\mathrm{QCD}} / m_{c, b}$ corrections. The dotted curves show the upper and lower bounds neglecting the order $\alpha_{s}$ corrections, but keeping the order $\Lambda_{\mathrm{QCD}} / m_{c, b}$ terms. The enhancement of the difference between the upper and lower bounds,

\footnotetext{
§It was first computed to order $\Delta^{2} / m_{c, b}^{2}$ in 113 .
} 


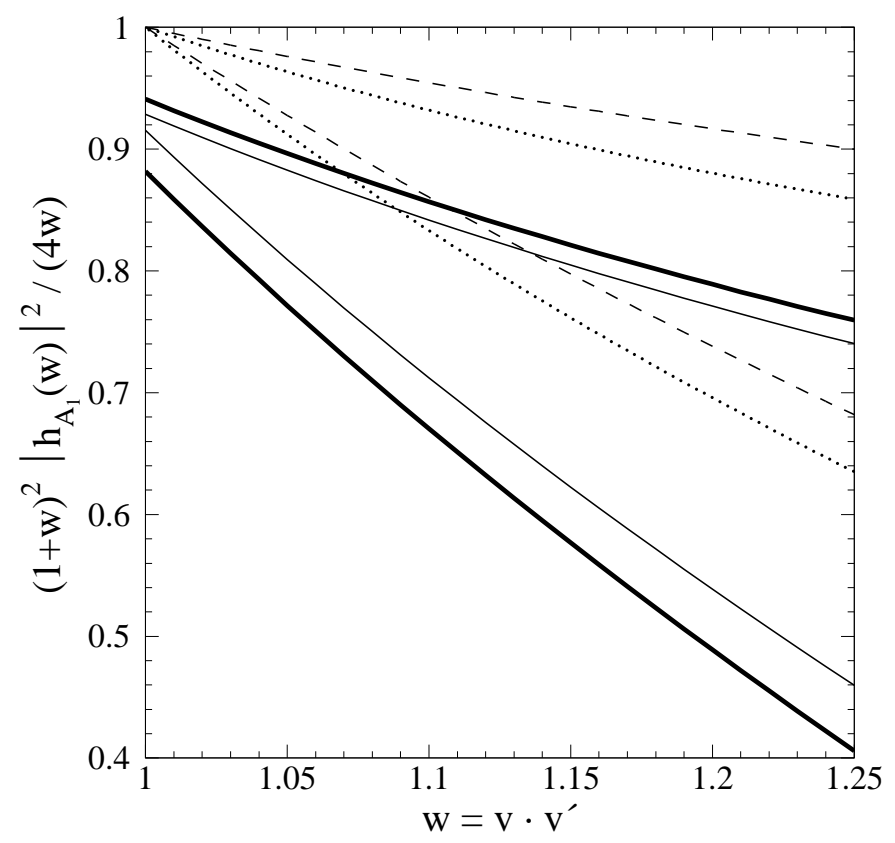

FIG. 3. Upper and lower bounds on $(1+w)^{2}\left|h_{A_{1}}(w)\right|^{2} /(4 w)$. The thin and thick solid curves are the bounds including effects of order $\alpha_{s}$ and $\Lambda_{\mathrm{QCD}} / m_{c, b}$, and corespond to $\Delta=1 \mathrm{GeV}$ and $2 \mathrm{GeV}$, respectively. The dashed curves are the bounds neglecting order $\alpha_{s}$ and $\Lambda_{\mathrm{QCD}} / m_{c, b}$ corrections. The dotted curves are the bounds neglecting order $\alpha_{s}$ but keeping $\Lambda_{\mathrm{QCD}} / m_{c, b}$ corrections.

which is seen to increase with $w$, is dominated by the perturbative corrections. The reason for the somewhat larger than usual deviation of

$$
\left|h_{A_{1}}(1)\right|^{2}=\eta_{A}^{2}=1-2 \frac{\alpha_{s}}{\pi}\left(\frac{1+z}{1-z} \ln z+\frac{8}{3}\right)+\ldots
$$

from unity in Fig. 3 is due to our choice of $\alpha_{s}=0.3$, which gives at order $\alpha_{s}, \eta_{A}=0.96$.

In plotting Fig. 3, we used $\lambda_{1}=-0.2 \mathrm{GeV}^{2}$ and $\lambda_{2}=0.12 \mathrm{GeV}^{2}$. While $\lambda_{2}$ is welldetermined by the $B^{*}-B$ mass splitting, the value of $\lambda_{1}$ is more uncertain. Changing $\lambda_{1}$ by $\pm 0.2 \mathrm{GeV}^{2}$ changes the lower bound at $w=1.25$ by $\mp 0.04$. (At order $\Lambda_{\mathrm{QCD}} / m_{c, b}$ the value of $\lambda_{1}$ does not affect the upper bound for all $w$, nor the lower bound at zero recoil.) We neglected the nonperturbative corrections of order $\Lambda_{\mathrm{QCD}}^{2} / m_{c, b}^{2}$. Such corrections to the lower bound involve matrix elements of dimension- 6 operators in the operator product expansion. The order $\Lambda_{\mathrm{QCD}}^{2} / m_{c, b}^{2}$ corrections to the upper bound [on the right-hand side of the first inequality in (22)] are given by 13, 15 


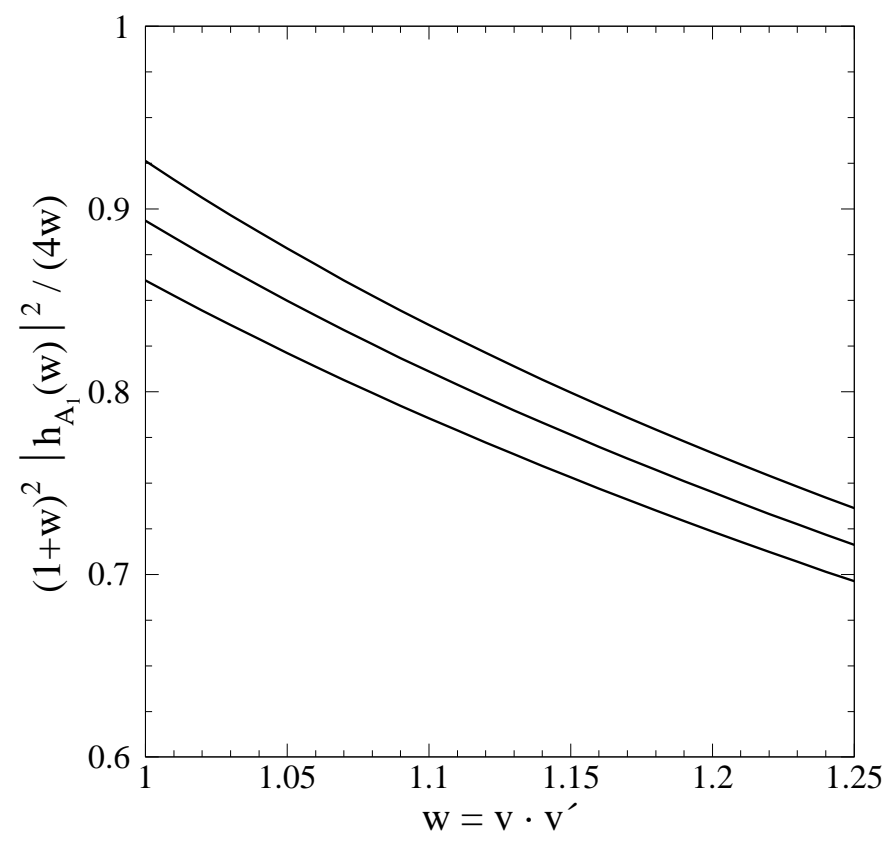

FIG. 4. Upper bound on $(1+w)^{2}\left|h_{A_{1}}(w)\right|^{2} /(4 w)$ including order $\Lambda_{\mathrm{QCD}}^{2} / m_{c, b}^{2}$ corrections for different values of $\lambda_{1}$. The curves from above to below correspond to $\lambda_{1}=0,-0.2 \mathrm{GeV}^{2}$, and $-0.4 \mathrm{GeV}^{2}$, respectively.

$$
\frac{w^{2}-1}{2 w} \frac{\left(\lambda_{1}-\lambda_{2}\right) w^{2}-\bar{\Lambda}^{2}\left(3-2 w^{2}\right)}{2 m_{c}^{2} w^{4}}+\frac{\lambda_{1}-\lambda_{2} w^{2}}{4 m_{c}^{2} w^{5}}+\frac{\lambda_{1}+3 \lambda_{2}}{12 m_{c}^{2} w^{3}}\left(3 w^{2} z^{2}+2 z\right) .
$$

For the central values of $\bar{\Lambda}$ and $\lambda_{1}$ used throughout this paper, these corrections affect the upper bounds by only about $-(0.02-0.03)$. The size of this correction is sensitive to the value of $\lambda_{1}$, while it is largely independent of $\bar{\Lambda}$. In Fig. 4 we plot the upper (Bjorken) bound on $\left|h_{A_{1}}(w)\right|^{2}$ including the order $\Lambda_{\mathrm{QCD}}^{2} / m_{c, b}^{2}$ corrections in eq. (28) for $\Delta=1 \mathrm{GeV}$ and three different values of $\lambda_{1}, \lambda_{1}=0,-0.2 \mathrm{GeV}^{2}$, and $-0.4 \mathrm{GeV}^{2}$. Changing $\bar{\Lambda}$ by $\pm 0.1 \mathrm{GeV}$ from the value we used (i.e., $0.4 \mathrm{GeV}$ ) affects the curves plotted in Fig. 4 by about $\mp 0.01$. If $\lambda_{1}$ is known more accurately in the future, this upper bound may constrain $h_{A_{1}}(1)$ to be somewhat below $\eta_{A}$.

A larger value of $\Delta$ increases our confidence in the validity of using perturbative QCD to evaluate the time ordered product of weak currents. In going from $\Delta=2 \mathrm{GeV}$ to $\Delta=3 \mathrm{GeV}$ the upper bound (the upper thick solid curve in Fig. 3) is increased by about 0.01 fairly independently of $w$ over the region $1<w<1.25$, while the lower bound (the lower thick 
solid curve in Fig. 3) is decreased by 0.06 at $w=1$ and by 0.07 at $w=1.25$. Clearly, the lower bound is considerably more sensitive to the choice of $\Delta$ than the upper bound.

Converting the bounds in eq. (22) into constraints on the slope parameter of the $h_{A_{1}}$ form factor at zero recoil, $\rho_{A_{1}}^{2}$, is not straightforward. The upper and lower bounds on $\left|h_{A_{1}}(w)\right|^{2}$ do not meet at zero recoil, and therefore a bound on $\rho_{A_{1}}^{2}$ can only be derived with some smoothness assumption. We bound $\rho_{A_{1}}^{2}$ by assuming that $h_{A_{1}}(w)$ is linear over the region $1<w<1.25$. Then the plots in Fig. 3 (which neglect terms of order $\Lambda_{\mathrm{QCD}}^{2} / m_{c, b}^{2}$ ) imply the bounds $0.44+4\left[h_{A_{1}}(1)-\eta_{A}\right]<\rho_{A_{1}}^{2}<1.19+4\left[h_{A_{1}}(1)-\eta_{A}\right]$ and $0.39+4\left[h_{A_{1}}(1)-\eta_{A}\right]<$ $\rho_{A_{1}}^{2}<1.36+4\left[h_{A_{1}}(1)-\eta_{A}\right]$ for $\Delta=1 \mathrm{GeV}$ and $2 \mathrm{GeV}$, respectively. Recall that $h_{A_{1}}(1)-\eta_{A}$ is of order $\Lambda_{\mathrm{QCD}}^{2} / m_{c, b}^{2}$. The increase in the lower bound compared to the $1 / 4$ at zeroth order is mostly due to the terms proportional to $\bar{\Lambda} / m_{c}$ in eqs. (22), and hence it is sensitive to the value of $\bar{\Lambda}$ we choose. It is interesting that this correction has the opposite sign than the QCD sum rule results in the effective theory, which predicted that order $\Lambda_{\mathrm{QCD}} / m_{c, b}$ corrections lower the values of the bounds.

The smoothness assumption used to derive bounds on the slope parameter $\rho_{A_{1}}^{2}$ from Fig. 3 can be justified model independently using the parameterization of Ref. [26]. This work also lets us extend the bounds at small $w$ presented in Fig. 3 to larger values of $w$. The $\Delta=2 \mathrm{GeV}$ bounds imply upper and lower bounds at $w=1.5$ of $0.86>h_{A_{1}}(1.5)>0.36$.

The bounds presented in this section can also be used for unpolarized $\Lambda_{b} \rightarrow \Lambda_{c} \ell \bar{\nu}$ decay if certain replacements are made. The form factors for the matrix element of the axial current, $G_{i}$, are defined by

$$
\left\langle\Lambda_{c}\left(v^{\prime}\right)\left|A^{\mu}\right| \Lambda_{b}(v)\right\rangle=\bar{u}\left(v^{\prime}\right)\left[G_{1} \gamma^{\mu}+G_{2} v^{\mu}+G_{3} v^{\prime \mu}\right] \gamma_{5} u(v) .
$$

Bounds on $G_{1}$ are obtained by replacing the left-hand side of eqs. (22) by $G_{1}^{2}(w+1) /(2 w)$. The quantities $\bar{\Lambda}, \lambda_{1}$, and $\lambda_{2}$ that appear on the right-hand side must now be interpreted as arising from $\Lambda_{b}$ matrix elements. They are simply related to the corresponding quantities in the $B$ meson case [27], $\lambda_{2}\left(\Lambda_{b}\right)=0$, 


$$
\begin{aligned}
\lambda_{1}\left(\Lambda_{b}\right) & =\lambda_{1}(B)+2 m_{c}\left[\left(m_{\Lambda_{b}}-\bar{m}_{B}\right)-\left(m_{\Lambda_{c}}-\bar{m}_{D}\right)\right] /(1-z), \\
\bar{\Lambda}\left(\Lambda_{b}\right) & =\bar{\Lambda}(B)+m_{\Lambda_{b}}-\bar{m}_{B}+\left[\lambda_{1}\left(\Lambda_{b}\right)-\lambda_{1}(B)\right] /\left(2 m_{b}\right) .
\end{aligned}
$$

\section{ORDER $\alpha_{s}^{2} \beta_{0}$ CORRECTIONS AT ZERO RECOIL}

At zero recoil we can rewrite the upper and lower bounds on the $h_{A_{1}}$ form factor in eq. (22) as

$$
\begin{aligned}
& \left|h_{A_{1}}(1)\right|^{2}<\eta_{A}^{2}+\frac{\alpha_{s}(\Delta)}{\pi} A_{1}+\frac{\alpha_{s}^{2}(\Delta)}{\pi^{2}} \beta_{0} A_{2}, \\
& \left|h_{A_{1}}(1)\right|^{2}>\eta_{A}^{2}+\frac{\alpha_{s}(\Delta)}{\pi} A_{1}+\frac{\alpha_{s}^{2}(\Delta)}{\pi^{2}} \beta_{0} A_{2}-\frac{\Delta}{m_{M_{1}}-m_{M}}\left[\frac{\alpha_{s}(\Delta)}{\pi} B_{1}+\frac{\alpha_{s}^{2}(\Delta)}{\pi^{2}} \beta_{0} B_{2}\right] .
\end{aligned}
$$

These bounds also receive nonperturbative corrections of order $\Lambda_{\mathrm{QCD}}^{2} / m_{c, b}^{2}$. Such corrections to the upper bound are given by

$$
-\frac{\lambda_{2}}{m_{c}^{2}}+\frac{\lambda_{1}+3 \lambda_{2}}{4 m_{c}^{2}}\left(1+\frac{2}{3} z+z^{2}\right)
$$

The order $\Lambda_{\mathrm{QCD}}^{2} / m_{c, b}^{2}$ corrections to the lower bound on $\left|h_{A_{1}}(1)\right|^{2}$ depend on additional quantities (besides $\lambda_{1}$ and $\lambda_{2}$ ) that parametrize matrix elements of dimension-6 operators in the operator product expansion.

The corrections in $\eta_{A}$ in eq. (31) arise, at the parton level, from the final state $X=c$. Except for an infrared renormalon ambiguity, $\eta_{A}$ only depends on physics associated with the scales $m_{c, b}$. It has been calculated to order $\alpha_{s}^{2} \beta_{0}$ [28]. Explicitly,

$$
\eta_{A}=1-\frac{\alpha_{s}\left(\sqrt{m_{b} m_{c}}\right)}{\pi}\left(\frac{1+z}{1-z} \ln z+\frac{8}{3}\right)-\frac{\alpha_{s}^{2}\left(\sqrt{m_{b} m_{c}}\right)}{\pi^{2}} \beta_{0} \frac{5}{24}\left(\frac{1+z}{1-z} \ln z+\frac{44}{15}\right) .
$$

The full order $\alpha_{s}^{2}$ expressions for $\eta_{A}$ and $\eta_{V}$ are also known approximately [29]. For $\eta_{A}$ the order $\alpha_{s}^{2} \beta_{0}$ terms dominate the $\alpha_{s}^{2}$ correction, while for $\eta_{V}$ they do not.

The corrections in $A_{i}$ and $B_{i}$ originate from final states $X$ that contain a charm quark plus additional partons, e.g., $c g, c \bar{q} q$, etc. These corrections are suppressed by powers of $\Delta / m_{c, b}$. For convenience, we evaluated the arguments of the strong couplings in eqs. (31) and (33) at two different scales $\left(\sqrt{m_{c} m_{b}}\right.$ for the series in $\eta_{A}$ and $\Delta$ for the other terms). 


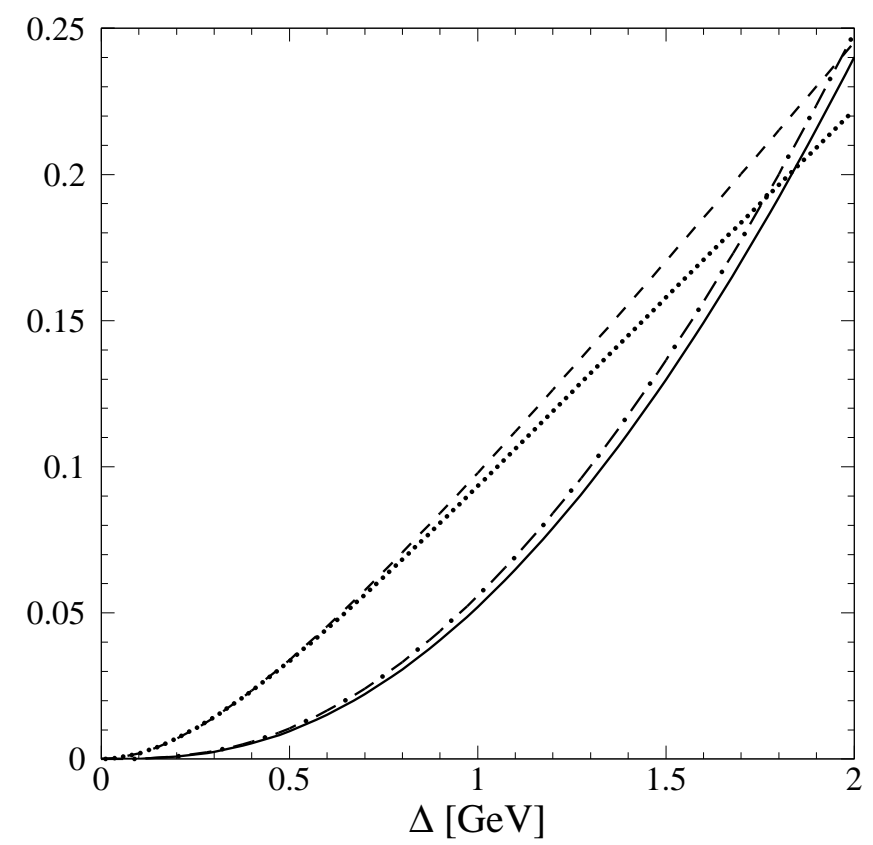

FIG. 5. The functions $A_{1}, B_{1}, A_{2}$, and $B_{2}$ defined in eq. (31) as a function of $\Delta$. The dotted curve is $A_{1}$, the dash-dotted curve is $B_{1}$, the dashed curve is $A_{2}$, and the solid curve is $B_{2}$.

Of course, it is possible to evaluate both series at the same scale using the QCD $\beta$-function [30]. The functions $A_{1}$ and $B_{1}$ are given in Sec. V, $A_{2}$ was computed in Ref. [14], our result for $B_{2}$ is given below. These order $\alpha_{s}^{2} \beta_{0}$ corrections are relatively simple to compute due to a relation between the $n_{f}$ dependent part of the order $\alpha_{s}^{2}$ terms and the order $\alpha_{s}$ result with a finite gluon mass [31]. The calculation is simplest in the so-called $V$-scheme, but we present the results in the usual $\overline{\mathrm{MS}}$ scheme. To leading order in $d=\Delta / m_{c}$

$$
\begin{aligned}
A_{1} & =\frac{d^{2}}{3}\left(1+\frac{2}{3} z+z^{2}\right), & A_{2} & =\frac{1}{2}\left(\frac{13}{6}-\ln 2\right) A_{1}+\frac{d^{2}}{15}\left(1+\frac{4}{3} z+z^{2}\right), \\
B_{1} & =\frac{2}{3} A_{1}, & B_{2} & =\frac{2}{3} A_{2}-\frac{d^{2}}{54}\left(1+\frac{2}{3} z+z^{2}\right) .
\end{aligned}
$$

To all orders in $d, A_{1}$ and $B_{1}$ are given in eq. (25), and $A_{2}$ and $B_{2}$ are computed numerically. In Fig. 5 we plot the functions $A_{1}, B_{1}, A_{2}$, and $B_{2}$ as a function of $\Delta$. The dotted curve is $A_{1}$, the dash-dotted curve is $B_{1}$, the dashed curve is $A_{2}$, and the solid curve is $B_{2}$.

On the right-hand sides of eqs. (31) the renormalon ambiguity of order $\Lambda_{\mathrm{QCD}}^{2} / m_{c, b}^{2}$ in $\eta_{A}^{2}$ is cancelled by that in the series $A_{i}$. Therefore, in considering the size of the $\alpha_{s}^{2}$ corrections relative to those of order $\alpha_{s}$, it seems reasonable for the upper bound to take all terms on the 
right-hand side of eqs. (31) together [32]. Although $A_{2}$ is approximately as big as $A_{1}$ (over the region $\Delta<2 \mathrm{GeV}$ ), since the order $\alpha_{s}^{2} \beta_{0}$ term in $\eta_{A}^{2}$ partially cancels against $A_{2}$, the perturbative expansion for the upper bound seems reasonably well-behaved.F For example, with $\Lambda_{\mathrm{QCD}}=200 \mathrm{MeV}$ and $\Delta=1 \mathrm{GeV}$, one finds neglecting terms of order $\Lambda_{\mathrm{QCD}}^{2} / m_{c, b}^{2}$ that

$$
\begin{array}{r}
\left|h_{A_{1}}(1)\right|^{2}<1-0.073-0.019 \\
+0.013+0.017 \\
=1-0.060-0.002 .
\end{array}
$$

The last two terms in the first line are the order $\alpha_{s}$ and $\alpha_{s}^{2} \beta_{0}$ corrections to $\eta_{A}^{2}$, while the second line contains the terms proportional to $A_{1}$ and $A_{2}$. For values of $\Delta$ between $1 \mathrm{GeV}$ and $5 \mathrm{GeV}$ the cancellation of order $\alpha_{s}^{2} \beta_{0}$ terms persists.

For the lower bound, the order $\alpha_{s}^{2} \beta_{0}$ term in the perturbative expansion of the term proportional to $\Delta /\left(m_{M_{1}}-m_{M}\right)$, which originates from the first moment of the time ordered product of weak currents, is about as large as the order $\alpha_{s}$ term over the region $\Delta<5 \mathrm{GeV}$. With $\Lambda_{\mathrm{QCD}}=200 \mathrm{MeV}$ and $\Delta=1 \mathrm{GeV}$, the lower bound in eq. (22) is

$$
\begin{aligned}
\left|h_{A_{1}}(1)\right|^{2}>1 & -0.060-0.002 \\
& -0.019-0.022 \\
= & 1-0.079-0.024 .
\end{aligned}
$$

The terms in the first line arise from $\eta_{A}^{2}$ and the series $A_{i}$, while the second line contains the terms proportional to $B_{1}$ and $B_{2}$. Note that the coefficient of the $B_{i}$ terms depend on the mass of the first excited state.

\footnotetext{
** Ref. [14] was less certain that the upper bound on $\left|h_{A_{1}}(1)\right|^{2}$ has a well-behaved perturbative expansion. This is mostly due to the fact that in [14] the behavior of the perturbative series arising from the terms $A_{i}$ (which contain all the $\Delta$-dependence) was discussed without combining it with that from $\eta_{A}^{2}$.
} 


\section{CONCLUSIONS}

In this paper we studied corrections to the Bjorken and Voloshin sum rules on form factors of semileptonic $B \rightarrow D^{(*)} \ell \bar{\nu}$ decays. In the heavy quark effective theory we derived upper and lower bounds on the Isgur-Wise function, and on its slope at zero recoil. Matching the full theory onto HQET, we translated the bounds in the effective theory into bounds on $\mathcal{F}_{B \rightarrow D^{(*)}}$, the shape of the measured $B \rightarrow D^{(*)}$ spectrum. The results in Table I show that while the corrections to the Bjorken bound (lower bound on $\rho_{B \rightarrow D^{(*)}}^{2}$ ) are small, the corrections to the Voloshin bound (upper bound on $\rho_{B \rightarrow D^{(*)}}^{2}$ ) are sizable. The reason is that perturbative corrections to the Voloshin bound are enhanced by $\Delta / \bar{\Lambda}$. Therefore, even if experimental data would settle around $\rho_{B \rightarrow D^{(*)}}$ slightly above unity, that would still not be a problem for the theory to accommodate.

The bounds derived in Sec. IV are affected at order $\Lambda_{\mathrm{QCD}} / m_{c, b}$ by corrections that are parametrized by four subleading universal functions, and are not known at present model independently. Therefore, we also studied the sum rule constraints on the $h_{A_{1}}(w)$ form factor in the full theory. In this approach, all $\Lambda_{\mathrm{QCD}} / m_{c b}$ corrections to the sum rules are parametrized by the three matrix elements, $\bar{\Lambda}, \lambda_{1}$, and $\lambda_{2}$. Bounds on the $h_{A_{1}}$ form factor are shown in Fig. 3, and with some assumptions we found that its slope parameter satisfies $0.4 \lesssim \rho_{A_{1}}^{2} \lesssim 1.3$

These bounds on $h_{A_{1}}$ in the full theory of QCD can also be related to bounds on $\rho_{B \rightarrow D^{(*)}}^{2}$. Using eqs. (17) and (19) we find that the order $\alpha_{s}$ corrections imply $\rho_{B \rightarrow D^{*}}^{2}=\rho_{A_{1}}^{2}-0.04$ and $\rho_{B \rightarrow D}^{2}=\rho_{A_{1}}^{2}+0.05$. These relations receive order $\Lambda_{\mathrm{QCD}} / m_{c, b}$ corrections. Due to heavy quark spin symmetry the order $\Lambda_{\mathrm{QCD}} / m_{c, b}$ difference between $\rho_{B \rightarrow D^{(*)}}^{2}$ and $\rho_{A_{1}}^{2}$ is independent of the subleading Isgur-Wise function $\chi_{1}$. Then eqs. (18) and (20), together with the QCD sum rule predictions for the subleading Isgur-Wise functions, imply that order $\Lambda_{\mathrm{QCD}} / m_{c, b}$ terms reduce $\rho_{B \rightarrow D^{*}}^{2}-\rho_{A_{1}}^{2}$ by 0.11 and reduce $\rho_{B \rightarrow D}^{2}-\rho_{A_{1}}^{2}$ by 0.01 . (The uncertainty in these predictions will be reduced if the form factor ratio $R_{2}$ can be measured precisely.)

One of the largest uncertainties in the sum rule predictions (especially in the Voloshin 
bound) is related to the numerical values of $\bar{\Lambda}$ and $m_{M_{1}}-m_{M}$. In this paper we used $m_{M_{1}}-$ $m_{M}=\bar{\Lambda}=0.4 \mathrm{GeV}$, motivated by the experimentally measured $D_{1}-D^{*}$ mass difference, and by the extraction of $\bar{\Lambda}$ in [33]. However, the uncertainties in this determination of $\bar{\Lambda}$ are sizable, and precise experimental data on other inclusive processes is needed to extract the value of this quantity more reliably [34]. Taking $m_{M_{1}}-m_{M}=m_{D_{1}}-m_{D^{*}}$ may also be misleading. Besides the possibility of sizable decay rates into non-resonant final states [35] (discussed in Sec. II), there is probably a doublet lighter than the $\left\{D_{1}, D_{2}^{*}\right\}$ heavy quark spin symmetry doublet, that is of order $100 \mathrm{MeV}$ or more broad. The spin-parity of the light degrees of freedom in the $\left\{D_{1}, D_{2}^{*}\right\}$ doublet is ${s_{l}}_{l}{ }^{\prime} \frac{3}{2}^{+}$, and so the $D_{1}$ is $1^{+}$, while the $D_{2}^{*}$ is $2^{+}$. However, light degrees of freedom with $s_{l}^{\pi_{l}}=\frac{1}{2}^{+}$yield a doublet of $0^{+}$and $1^{+}$states. These can decay into $D^{(*)} \pi$ in an $s$-wave, and so they should be much broader than the $\left\{D_{1}, D_{2}^{*}\right\}$ that can only decay in a $d$-wave. (An $s$-wave decay amplitude for the $D_{1}$ is allowed by angular momentum conservation, but it is forbidden by heavy quark spin symmetry [36].) A reduction in $m_{M_{1}}-m_{M}$ would further weaken the Voloshin bound.

Some improvements in this paper are possible. We focused on the region near zero recoil because it is important for the extraction of $\left|V_{c b}\right|$. It would be straightforward to calculate the terms in eq. (22) of order $\alpha_{s}(w-1)^{2}$. Then Fig. 3 could be extended over the full kinematic range $1<w<1.5$. Some uncertainty in the sum rules arises from the order $\alpha_{s}^{2}$ corrections. The part of these corrections proportional to the one-loop $\beta$-function have been computed at zero recoil. For the part of the lower bound involving the first moment of the time ordered product of weak currents these corrections are as big as the order $\alpha_{s}$ corrections, unless $\Delta$ is quite large. It should be possible to compute the $\alpha_{s}^{2} \beta_{0}$ corrections away from zero recoil, and also to the bounds on $\rho_{B \rightarrow D^{(*)}}^{2}$ considered in Section III.

\section{ACKNOWLEDGMENTS}

We would like to thank B. Grinstein for critical reading of the manuscript. C.G.B. thanks L. Wolfenstein for stimulating conversations, and the nuclear theory group at University 
of Washington for their hospitality during the completion of this work. This work was supported in part by the U.S. Dept. of Energy under Grant no. DE-FG03-92-ER 40701, DE-FG02-91ER40682, and DOE-FG03-90ER40546. 


\section{REFERENCES}

[1] N. Isgur and M.B. Wise, Phys. Lett. B232 (1989) 113; Phys. Lett. B237 (1990) 527.

[2] S. Nussinov and W. Wetzel, Phys. Rev. D36 (1987) 130.

[3] M. Voloshin and M. Shifman, Sov. J. Nucl. Phys. 47 (1988) 511.

[4] M.E. Luke, Phys. Lett. B252 (1990) 447.

[5] J. Chay, H. Georgi and B. Grinstein, Phys. Lett. B247 (1990) 399; M. Voloshin and M. Shifman, Sov. J. Nucl. Phys. 41 (1985) 120.

[6] M. Luke, M.J. Savage, and M.B. Wise, Phys. Lett. B343 (1995) 329; Phys. Lett. B345 (1995) 301.

[7] J.D. Bjorken, Invited talk given at Les Rencontre de la Valle d'Aoste (La Thuile, Italy), SLAC-PUB-5278 (1990); J.D. Bjorken, I. Dunietz, J. Taron, Nucl. Phys. B371 (1992) 111.

[8] N. Isgur and M.B. Wise, Phys. Rev. D43 (1991) 819.

[9] M.B. Voloshin, Phys. Rev. D46 (1992) 3062.

[10] T. Bergfeld et al., CLEO Collaboration, CLEO CONF 96-3, ICHEP96 PA05-078; B. Barish et al., CLEO Collaboration, Phys. Rev. D51 (1995) 1014.

[11] ALEPH Collaboration, PA05-056, Paper contributed to the ICHEP96 Conference.

[12] A. Anastassov et al., CLEO Collaboration, CLEO CONF 96-8, ICHEP96 PA05-079.

[13] I.I. Bigi, M.A. Shifman, N.G. Uraltsev, and A.I. Vainshtein, Phys. Rev. D52 (1995) 196.

[14] A. Kapustin, Z. Ligeti, M.B. Wise and B. Grinstein, Phys. Lett. B375 (1996) 327.

[15] C.G. Boyd and I.Z. Rothstein, UCSD-TH-96-20 hep-ph/9607418].

[16] E.C. Poggio, H.R. Quinn, and S. Weinberg, Phys. Rev. D13 (1976) 1958.

[17] E. Eichten and B. Hill, Phys. Lett. B234 (1990) 511; H. Georgi, Phys. Lett. B240 (1990) 447.

[18] A.G. Grozin and G.P. Korchemsky, Phys. Rev. D53 (1996) 1378.

[19] G.P. Korchemsky and M. Neubert (unpublished); M. Neubert, Int. J. Mod. Phys. A11 (1996) 4173

[20] I. Bigi, A.G. Grozin, M. Shifman, N.G. Uraltsev, and A. Vainshtein, Phys. Lett. B339 (1994) 160.

[21] C.G. Boyd, B. Grinstein, A.V. Manohar, Phys. Rev. D54 (1996) 2081. 
[22] A.F. Falk, H. Georgi, B. Grinstein, and M.B. Wise, Nucl. Phys. B343 (1990) 1; A.F. Falk and B. Grinstein, Phys. Lett. B247 (1990) 406; M. Neubert, Phys. Rev. D46 (1992) 2212.

[23] M. Neubert, Phys. Rept. 245 (1994) 259.

[24] M. Neubert, Phys. Rev. D46 (1992) 3914; M. Neubert, Z. Ligeti and Y. Nir, Phys. Lett. B301 (1993) 101; Phys. Rev. D47 (1993) 5060; Z. Ligeti, Y. Nir and M. Neubert, Phys. Rev. D49 (1994) 1302.

[25] I. Caprini and M. Neubert, Phys. Lett. B380 (1996) 376.

[26] C.G. Boyd, B. Grinstein, R.F. Lebed, Phys. Lett. B353 (1995) 306; Nucl. Phys. B461 (1996) 493; C.G. Boyd and R.F. Lebed, UCSD-PTH-95-23 hep-ph/9512363, to appear in Nucl. Phys. B.

[27] A.V. Manohar and M.B. Wise, Phys. Rev. D49 (1994) 1310.

[28] M. Neubert, Phys. Lett. B341 (1995) 367.

[29] A. Czarnecki, Phys. Rev. Lett. 76 (1996) 4124.

[30] H.D. Politzer, Phys. Rev. Lett. 30 (1973) 1346; D.J. Gross and F. Wilczek, Phys. Rev. Lett. 30 (1973) 1343.

[31] B.H. Smith and M.B. Voloshin, Phys. Lett. B340 (1994) 176.

[32] We thank M. Shifman and N. Uraltsev for emphasizing this to us; see also N. Uraltsev, UND-HEP-96-BIG 04 hep-ph/9610425.

[33] M. Gremm, A. Kapustin, Z. Ligeti and M.B. Wise, Phys. Rev. Lett. 77 (1996) 20.

[34] Z. Ligeti and Y. Nir, Phys. Rev. D49 (1994) 4331; A. Kapustin and Z. Ligeti, Phys. Lett. B355 (1995) 318; A.F. Falk, M. Luke, and M.J. Savage, Phys. Rev. D53 (1996) 6316 .

[35] For a recent discussion of semileptonic $B$ decays into non-resonant final states, see: N. Isgur, JLAB-TH-96-16 [hep-ph/9610277].

[36] N. Isgur, M.B. Wise, Phys. Rev. Lett. 66 (1991) 1130. 